\title{
Cerebral correlates of declarative memory dysfunctions in early traumatic brain injury
}

\author{
J M Serra-Grabulosa, C Junqué, K Verger, P Salgado-Pineda, C Mañeru, J M Mercader
}

J Neurol Neurosurg Psychiatry 2005;76:129-131. doi: 10.1136/jnnp.2004.027631

We investigated residual brain damage in subjects who suffered severe traumatic brain injury (TBI) in childhood, and its relationship with declarative memory impairment. Magnetic resonance imaging (MRI) volumetric data and memory performance were compared between 16 adolescents with antecedents of severe TBI and 16 matched normal controls. Volumes of grey matter, white matter, cerebrospinal fluid (CSF), hippocampus, and caudate nuclei were measured. Verbal memory was assessed by the Rey's Auditory Verbal Learning test and visual memory by the Rey's Complex Figure. TBI patients performed significantly worse than controls in both verbal and visual memory. Patients presented decreased white matter volume and increased CSF. The hippocampus was reduced, but not the caudate nuclei. Memory performance correlated with CSF. Plasticity is incomplete for structural and functional deficits in children with TBI. Hippocampal atrophy, white matter loss, and memory impairment remain until adolescence. Memory sequelae are related more to diffuse brain injury, as reflected by MRI findings of increased CSF, than to hippocampal injury.

O ne of the most important cognitive sequelae of traumatic brain injury (TBI) is memory impairment. Post-mortem neuropathological studies, ${ }^{2}$ neuroimaging findings, ${ }^{3}{ }^{4}$ and data from animal models ${ }^{56}$ have shown that the hippocampus is highly susceptible to the effects of TBI. It seems plausible that the damage to this structure may be responsible for memory impairment in TBI subjects. Several studies have found correlations between memory impairment and hippocampal atrophy. ${ }^{3}$ However, learning deficits in TBI have also been related to striatal reductions ${ }^{7}$ and fornix damage. ${ }^{8}$ In children, memory deficits have been explained by frontal lobe damage. ${ }^{9}$

In the only volumetric study of hippocampal atrophy in paediatric TBI published to date, ${ }^{9}$ the authors reported negative findings. As the immature brain is more plastic than the adult, and as recent data suggest that the hippocampus is a region with neurogenesis, ${ }^{11}{ }^{11}$ it may be that hippocampal damage in TBI may be compensated for to a greater extent if the initial injury occurs during childhood rather than later in life.

The purpose of this study was to investigate the cerebral correlates of memory impairment in adolescents who suffered severe traumatic closed brain injury in childhood.

\section{METHODS}

Subjects

The TBI sample consisted of 16 subjects ( 14 boys and 2 girls). The patients were taken from a TBI subsample of patients described elsewhere, ${ }^{12}{ }^{13}$ who did not present massive focal lesions. None of the subjects had focal lesions greater than $8.5 \mathrm{~cm}^{3}$ measured by Analyze software (version 3.0) routines. Mean (SD) age of subjects at time of study was 17.88 (2.85) years, age at injury 8.18 (3.65) years, time since injury 9.68 (1.88) years, Glasgow Coma Scale 5.63 (1.78), and coma length 161.25 (132.09) hours. Hypoxia was reported in nine subjects. The control group consisted of 16 subjects matched to patients according to gender ( 14 boys and two girls), age (16.94 (3.21) years), length of education (11.25 (2.84) years), and parents' socioeconomic status. All subjects or their parents gave their informed consent and the study was approved by the local ethics committee.

\section{Neuropsychological assessment}

Verbal learning was evaluated using a version of Rey's Auditory Verbal Learning Test (RAVLT) and visual memory by Rey's Complex Figure (RCF). ${ }^{14}$ The measures used were: verbal learning (total number of words remembered in trial, $1-5$ ), verbal long term recall (number of words recalled after a 15 minute interval) and visual recall (3 minutes after copying the RCF).

\section{Magnetic resonance imaging study}

All scans were performed using a General Electric Signa 1.5 Tesla scanner (Milwaukee, WI, USA). A set of coronal images was acquired with a FSPGR 3D sequence (repetition time 12 seconds, echo time 5.2 seconds; TI 3001 nex; field of view $24 \times 24$; and $256 \times 256$ matrix. This sequence provides a voxel of $1.2 \times 0.9 \times 0.9 \mathrm{~mm}^{3}$.

We obtained 124 contiguous slices. The image data sets were processed on a Sun Solaris Ultra 60 workstation (Sun Microsystems Inc.) with the Analyze software (version 3.0; Mayo Foundation, Rochester, MN, USA). Firstly, the images were resized with the cubic force option to obtain an isotropic voxel $\left(1.2 \times 1.2 \times 1.2 \mathrm{~mm}^{3}\right)$. Tl weighted images were processed with the region of interest (ROI) feature of the Analyze program. ROI tracing was drawn manually. The volume measures were then quantified automatically. A trained operator who was blinded to the subjects' characteristics traced all measures. Intrarater reliability for volumetric measures was established by performing two analyses of 10 images of another sample, and then calculating the intrarater reliability coefficient, which was initially 0.84 . After evaluating the differences, 10 more images were measured; the intrarater reliability coefficient was 0.94 for the hippocampus and 0.95 for the caudate nucleus. For the regional measurements of the hippocampus and caudate nucleus we followed the procedures described by Bigler et $a l^{3}$ and Gunning-Dixon et al. ${ }^{15}$

Frontal lobe volumes were delimited from $\mathrm{T} 2$ weighted MRI axial slices by using the region of interest tool of the

Abbreviations: CSF, cerebrospinal fluid; MRI, magnetic resonance imaging; RAVLT, Rey's Auditory Verbal Learning Test; RCF, Rey's Complex Figure; TBI, traumatic brain injury 
MRIcro software (Nottingham, UK), creating an individual frontal mask, which was applied to the images. The basal portion of the lateral fissure delimited inferior-posterior boundaries of the frontal lobes. The presence of the basal ganglia or the frontal horn of the lateral ventricles defined medial surface. Normalised frontal brain images were then automatically segmented into separate images representing probability maps for frontal grey matter, white matter, and CSF using the combined pixel intensity and a priori knowledge approach integrated in the SPM99 software (Wellcome Department of Cognitive Neurology, University College, London, UK), and supplemented by the "lots of inhomogeneity corrections" option. We used an automatic MatLab routine to obtain volumetric measurements of the three frontal tissue compartments mentioned above. Regional brain volumes were corrected for brain size following the covariance estimate method proposed by Jack et al and Free et al. ${ }^{16}{ }^{17}$ Finally, the intracranial volume was obtained using the automatic image processing approach integrated in SPM99. We used an automatic MatLab routine to obtain volumetric measurements of the aforementioned three tissue compartments. Total brain measures were obtained by adding the values obtained from white matter, grey matter, and CSF values. The automatic segmentation process used in the present report comprises the whole brain including the cerebellum and the brainstem.

\section{RESULTS}

As all the neuropsychological and MRI variables were normally distributed, we used parametric test: two tailed $t$ test for groups comparisons and Pearson's test for correlation analysis. Bonferroni correction for multiple comparisons was not applied because the exploratory nature of the study and the reduced sample size examined.

TBI subjects performed worse than controls in both visual and verbal learning. We observed a bilateral hippocampal reduction in TBI subjects (fig l) but not in the caudate nucleus. Moreover, TBI subjects had global brain white matter volume atrophy and frontal white matter atrophy. We also observed CSF volume increase (see table).

Correlation analysis showed that verbal long term recall significantly correlated with CSF $(\mathrm{r}=-0.54 ; \mathrm{p}=0.029)$. The hippocampal volume tended to correlate with visual and verbal long term recall $(\mathrm{r}=0.45, \mathrm{p}=0.07 ; \mathrm{r}=0.43, \mathrm{p}=0.097$, respectively). Control subjects did not show any significant correlation.

\section{DISCUSSION}

The purpose of our study was to evaluate the long term brain damage of childhood TBI and the relationship of its effects

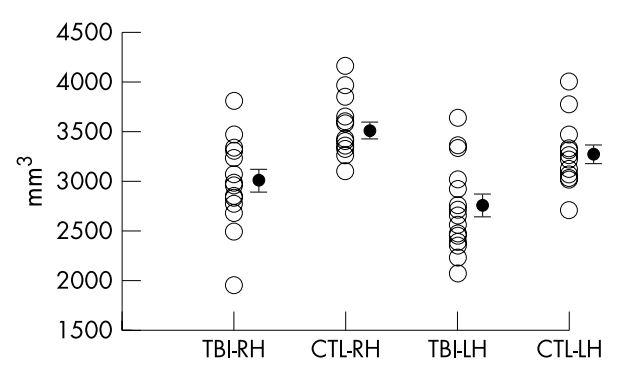

Figure 1 Right $(\mathrm{RH})$ and left $(\mathrm{LH})$ hippocampal volumes for each subject. Black vertical bars indicate volume means (SE). CTL, control subjects; TBI, traumatic brain injury subjects.

with declarative memory outcome. Our results showed that TBI subjects had clear hippocampal atrophy but that the caudate nuclei remained intact. We also obtained significant between group differences in measures of global subcortical atrophy such as CSF and white matter, and also in frontal white matter.

To our knowledge, this is the first study that demonstrates the persistence of hippocampal atrophy in children with TBI. Previously, Di Stefano et al had not shown differences in hippocampal volume between 25 children with severe TBI and 25 with mild TBI. The discrepancies in these results may be due to differences in the age at injury, time since injury, methods for MRI measurements, or characteristics of the control group. However, the presence of hippocampal atrophy on MRI in our study is in agreement with previous neuropathological findings in children with fatal $\mathrm{TBI}^{2}{ }^{18}$ and the findings of adults with TBI. ${ }^{3}$

Interestingly, despite plasticity mechanisms such as maturation of hippocampal formation from childhood to adulthood, $^{19}$ and hippocampal neurogenesis and glial proliferation, ${ }^{10}{ }^{11}$ our sample showed clear hippocampal atrophy after long term evolution from TBI. These results indicate that plasticity does not completely compensate for hippocampal neuronal loss in severe TBI.

As in previous investigations in both children and adults we found white matter volume decrease, ${ }^{821}$ CSF increase, ${ }^{820}$ hippocampal atrophy, ${ }^{34}$ and no differences in caudate nucleus size. ${ }^{7}$ Our results corroborate previous findings that the hippocampus is more sensitive to TBI effects than the striatum.

In the neuropsychological assessment, we also observed persistent memory impairment, a finding that challenges previous reports of the reversibility of cognitive impairment in children. ${ }^{12} 132022$ In the correlation analysis performed to identify the MRI parameter related to this deficit, the only

Table 1 Neuropsychological and volumetric $\left(\mathrm{mm}^{3}\right)$ measures in control and TBI subjects

\begin{tabular}{lcrrr}
\hline & Control, mean (SD) & TBI, mean (SD) & $t$ & P value \\
\hline Memory & & & & \\
$\quad$ Visual long term recall & $24.31(4.00)$ & $19.66(5.92)$ & -2.60 & 0.014 \\
$\quad$ Verbal learning & $56.25(5.71)$ & $40.81(9.03)$ & -5.77 & $<0.001$ \\
$\quad$ Verbal long term recall & $12.94(1.81)$ & $8.31(2.80)$ & -5.55 & $<0.001$ \\
Volumetric measures & & & & \\
$\quad$ Left hippocampus & $3259.08(302.05)$ & $2728.75(437.37)$ & -3.99 & $<0.001$ \\
Right hippocampus & $3504.19(283.11)$ & $3005.43(441.42)$ & -3.80 & 0.001 \\
Left caudate nucleus & $3922.43(489.32)$ & $3989.52(576.21)$ & 0.35 & 0.725 \\
Right caudate nucleus & $3971.99(490.08)$ & $4076.62(763.24)$ & 0.46 & 0.648 \\
White matter & $375271.02(17952.47)$ & $351823.08(19006.44)$ & -3.58 & 0.001 \\
Grey matter & $487612.76(26266.89)$ & $493991.11(22253.52)$ & 0.741 & 0.464 \\
CSF & $137116.22(14341.88)$ & $154185.81(15938.10)$ & 3.18 & 0.003 \\
Frontal white matter & $128686.70(4945.28)$ & $121870.21(5736.44)$ & -3.60 & 0.001 \\
Frontal grey matter & $149049.56(9472.24)$ & $150108.92(8100.71)$ & 0.34 & 0.736 \\
Frontal CSF & $47378.83(7246.01)$ & $49605.89(7290.31)$ & 0.86 & 0.393 \\
\hline CSF, cerebrospinal fluid & & & & \\
\hline
\end{tabular}


variable to reach significance was CSF, a measure of global atrophy. The CSF increase may be due to either white or grey matter loss, and both of these may be related to learning deficits, in addition to the possible role of the hippocampal damage. However, this correlation should be interpreted with caution, as we did not use the Bonferroni correction for multiple comparisons.

As regards the hippocampus, we only observed a trend towards significance in the correlation analysis. Because of the limbic circuitry involving hippocampal output, a number of white matter pathways may be damaged in traumatic brain injury and may be responsible for TBI memory sequelae. In addition to the diffuse white matter volume loss, there is probably white matter loss in the fornix, mammillothalamic track, and anterior thalamus projections to cortex and cingulate gyrus, which may be just as disruptive to memory as a specific hippocampal volume loss. ${ }^{4}$

Finally, this study has several limitations. The sample size precluded a regression analysis searching for contributors to memory loss. We did not measure the fornix, which may be involved in memory impairment in childhood TBI, ${ }^{4}$ and the neuroimaging tool we used to perform the brain segmentations, SPM, has several limitations in brain damaged subjects. ${ }^{23}$ Nonetheless, we think that the results obtained can contribute to increasing the knowledge of the brainbehaviour relationship in TBI occurring in the immature brain.

\section{ACKNOWLEDGMENTS}

This study was supported by grants from the Spanish Ministry of Education and Culture (DGICYT PM-98-0192), and the General Direction of Research from the Generalitat de Catalunya (1999SGR00081 and 2001SGR00139).

\section{Authors' affiliations \\ J M Serra-Grabulosa, C Junqué, K Verger, P Salgado-Pineda, \\ C Mañeru, Department of Psychiatry and Clinical Psychobiology.}

University of Barcelona, Spain

J M Serra-Grabulosa, C Junqué, J M Mercader, Institut d'Investigacions Biomèdiques August Pi i Sunyer (IDIBAPS), Barcelona, Spain

J M Mercader, Centre de Diagnòstic per la Imatge (CDI), Hospital Clínic de Barcelona, Barcelona

Competing interests: none declared

Correspondence to: Dr. Josep M Serra-Grabulosa, Department of Psychiatry and Clinical Psychobiology, University of Barcelona, Pg. Vall Hebron 171, 08035 Barcelona, Spain; imserra@ub.edu

Received 9 September 2003

In revised form 21 April 2004

Accepted 22 April 2004

\section{REFERENCES}

1 Bigler, ed. Brain imaging and behavioral outcome in traumatic brain injury. $J$ Learn Dis 1996;29:515-30.

2 Kotapka MJ, Graham DI, Adams JH, et al. Hippocampal damage in fatal paediatric head injury. Neuropath Appl Neurol 1993;19:128-33.

3 Bigler ED, Blatter DD, Anderson CV, et al. Hippocampal volume in normal aging and traumatic brain injury. Am J Neuroradiol 1997; 18:11-23.

4 Tate DF, Bigler, eds. Fornix and hippocampal atrophy in traumatic brain injury. Learn Memory 2000;7:442-6.

5 Kotapka MJ, Gennarelli TA, Graham DI, et al. Selective vulnerability of hippocampal neurons in acceleration-induced experimental head injury. J Neurotraum 1991:8:247-58.

6 Hicks RR, Smith DH, Lowenstain DH, et al. Mild experimental brain injury in the rat induces cognitive deficits associated with regional neuronal loss in the hippocampus. J Neurotraum 1993;10:405-14.

7 Primus EA, Bigler ED, Anderson CV, et al. Corpus striatum and traumatic brain injury. Brain Injury 1997; 11:577-86.

8 Gale SD, Johnson SC, Bigler ED, et al. Nonspecific white matter degeneration following traumatic brain injury. J Int Neuropsych Soc 1995; 1:17-28.

9 Di Stefano G, Bachevalier J, Levin HS, et al. Volume of focal brain lesions and hippocampal formation in relation to memory function after closed head injury in children. J Neurol Neurosurg Psych 2000;69:210-16.

10 Eriksson PS, Perfilieva E, Bjork-Eriksson T, et al. Neurogenesis in the adult human hippocampus. Nat Med 1998;4:1313-17.

11 Gould E, Reeves AJ, Fallah M, et al. Hippocampal neurogenesis in adult old world primates. Proc Natl Acad Sci USA 1999;96:5263-7.

12 Verger K, Junqué C, Jurado MA, et al. Age effects on long-term neuropsychological outcome in paediatric traumatic brain injury. Brain Injury 2000;14:495-503.

13 Verger $\mathrm{K}$, Junqué $\mathrm{C}$, Levin $\mathrm{H}$, et al. Correlation of atrophy measures on MRI with neuropsychological sequelae in children and adolescents with traumatic brain injury. Brain Injury 2001;15:211-21.

14 Lezak M. Neuropsychological Assessment. Oxford: Oxford University Press, 1995.

15 Gunning-Dixon FM, Head D, McQuain J, et al. Differential aging of the human striatum: a prospective MR imaging study. Am J Neuroradiol 1998;19:1501-7.

16 Jack CR, Tworney CK, Zinsmeister AR, et al. Anterior temporal lobes and hippocampal formations: normative volumetric measurements from MR images in young adults. Radiology 1989;172:549-54.

17 Free SL, Bergin PS, Fish DR, et al. Methods for normalization of hippocampal volumes measured with MR. Am J Neuroradiol 1995; 16:637-43.

18 Kotapka MJ, Graham DI, Adams JH, et al. Hippocampal pathology in fatal human head injury without high intracranial pressure. J Neurotraum 1994; 11:317-24.

19 Benes FM, Turtle M, Khan Y, et al. Myelination of a key relay zone in the hippocampal formation occurs in the human brain during childhood, adolescence, and adulthood. Arch Gen Psychiat 1994;51:477-84.

20 Berryhill P, Lilly MA, Levin HS, et al. Frontal lobe changes after severe diffuse closed head injury in children: a volumetric study of magnetic resonance imaging. Neurosurgery 1995;37:392-400.

21 Blatter DD, Bigler ED, Gale SD, et al. MR-based brain and cerebrospinal fluid measurement after traumatic brain injury: correlation with neuropsychological outcome. Am J Neuroradiol 1997; 18:1-10.

22 Levin HS, Culhane KA, Mendelsohn D, et al. Cognition in relation to magnetic resonance imaging in head-injured children and adolescents. Arch Neurol 1993;50:897-905.

23 Good CD, Johnsrude IS, Ashburner J, et al. A voxel-based morphometric study of ageing in 465 normal adult human brains. Neuroimage $2001 ; 14: 21-36$. 